\title{
Deformable Porous Media With Double Porosity III: Acoustics
}

\author{
J. L. AURIAULT \\ Sols Solides Structures, IMG, URA 1511, Université Joseph Fourier, Institut National Polytechnique de \\ Grenoble, CNRS, BP 53 X, 38041 Grenoble Cedex, France
}

and

\section{BOUTIN}

Ecole Nationale des Trataux Publics de l'Etat, Laboratoire Géomatériaux, rue M. Audin, 69518 Vaulx en Velin Cedex, France

\begin{abstract}
We investigate the acoustics of saturated porous media with a double porosity constituted by pores and fractures. This work is the direct extension of earlier papers by Auriault and Boutin, where the quasi-static behaviour was studied. The different macroscopic descriptions of the acoustics are shown to be the quasi-static ones, completed by classical inertial terms and with a generalized seepage law for the fractures. Therefore, when the three scales, i.e. the pore, the fracture and the macroscopic scales are equally separated, the medium exhibits memory effects. Finally, we investigate the interpretation of laboratory experiments on single porosity medium under an acoustic excitation. It is shown that the viscoelastic effects which are observed when the frequency is about a few $\mathrm{kHz}$ have their origins in the same phenomenon. But the macroscopic description now depends on the size and the shape of the sample, and therefore it is nonspecific for the porous medium.
\end{abstract}

Key words. Double porosity, acoustics.

\section{Nomenclature}

a elastic tensor of the skeleton material

$\mathbf{C}^{\prime}, \mathbf{c}^{\prime \prime} \quad$ effective elastic tensors of the skeleton

D rate of deformation tensor

e deformation tensor

f subscript for the fractures

$\mathbf{k}_{p}, \mathbf{k}_{f} \quad$ particular solutions for the velocity

field $v$ in the pores and the fractures, respectively

$\mathbf{K}_{p}, \mathbf{K}_{f} \quad$ filtration tensors of the pores and the fractures, respectively

I unit tensor

$l, l^{\prime}, l^{\prime \prime} \quad$ characteristic lengths of the pore scale, the fracture scale and the macroscopic medium, respectively

$n, n^{\prime} \quad$ pore porosity and fracture porosity,

N normal unit vector

$p_{p}, p_{f} \quad$ pressures in the pores and the fractures, respectively

p subscript for the pores

$Q \quad$ dimensionless number

Q $\quad$ quality factor

$s \quad$ subscript for the solid

$T_{n}, T_{f} \quad$ characteristic times for the pore and fracture flows, respectively

$\mathbf{u}_{s}, \mathbf{u}_{p}, \mathbf{u}_{f} \quad$ solid, pore and fracture fluid displacements, respectively

$\mathbf{v}_{s}, \mathbf{v}_{p}, \mathbf{v}_{f} \quad$ solid, pore and fracture fluid velocities, respectively

$\mathbf{x}, \mathbf{x}^{\prime}, \mathbf{x}^{\prime \prime}$ space variables for the pore, fracture and macroscopic scales, respectrespectively 
Greek

$\alpha^{\prime}, \alpha^{\prime \prime}, \gamma^{\prime}, \gamma^{\prime}$

coupling effective tensors entering the behavior relations of the porous medium

$\beta^{\prime}, \beta^{\prime \prime}$

$\Gamma, \Gamma^{\prime}$

$\Delta \quad$ Laplace operator

$\nabla \quad$ gradient operator

$\varepsilon \quad$ small parameter of the homogenization process

$\eta^{\prime} \quad$ particular solutions for the displacement field

$v^{\prime} \quad$ particular solutions for the pressure field

$\omega \quad$ pulsation

$\rho_{s}, \rho_{F} \quad$ density of the solid and the fluid, respectively

$\mu \quad$ viscosity

$\xi^{\prime} \quad$ particular solutions for the displace-

ment field $\sigma_{s}, \sigma_{p}, \sigma_{f} \quad$ solid, pore and fracture fluid stress tensors, respectively

$\tau^{\prime} \quad$ particular solutions for the pressure field

$\Omega, \Omega^{\prime} \quad$ periods at the pore and fracture scales, respectively

$\Omega_{s}, \Omega_{p}, \Omega_{s p}^{\prime}$, solid, pore and fracture fluid stress tensors, respectively

$\tau^{\prime} \quad$ particular solutions for the pressure field

$\Omega, \Omega^{\prime} \quad$ periods at the pore and fracture scales, respectively

$\Omega_{s}, \Omega_{p}, \Omega_{s p}^{\prime}, \Omega_{f}^{\prime}$ parts of the periods occupied by the solid, the pores, the solid plus the pores and the fractures, respectively

Particular Symbols

$\langle\Phi\rangle_{\Omega},\langle\Phi\rangle_{\Omega^{\prime}},\langle\langle\Phi\rangle\rangle_{\Omega \Omega^{\prime}}$ volume averages of the quantity $\Phi$ on $\Omega, \Omega^{\prime}$, and $\Omega$ plus $\Omega^{\prime}$, respectively.

\section{Introduction}

As in papers I and II (Auriault and Boutin, 1992, 1993), we consider porous saturated media of double porosity, i.e. where the porosity system is composed of two connected subsystems, pores and fractures, with very different characteristic lengths. Denoting $l$ and $l^{\prime}$ these two lengths, respectively, the porous media exhibits a small dimensionless parameter $l / l^{\prime} \ll 1$.

The geometry of the medium is recalled in Figures 1 and 2. For simplicity the two porosity subsystems are assumed as periodic. As pointed out in Auriault (1991a), the simplification is of no consequence for the structure of the macroscopic description. A random medium and a periodic one exhibit an identical macroscopic behaviour

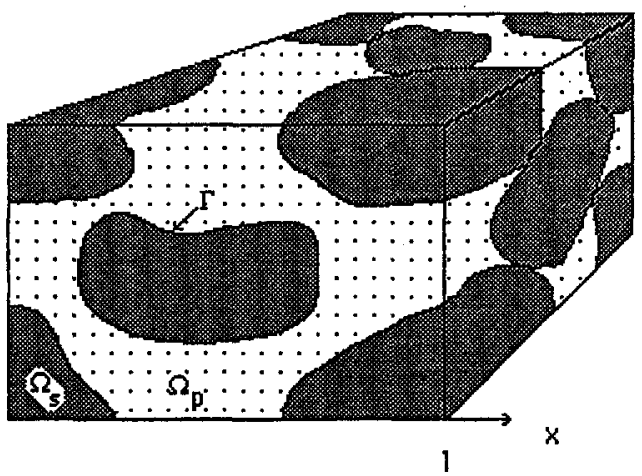

Fig. 1. Periodic cell of the microporous medium. 


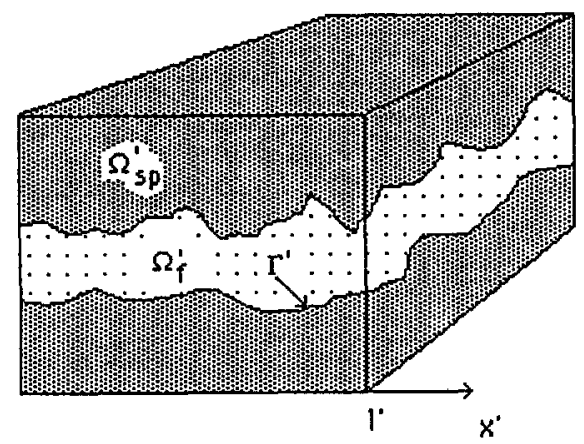

Fig. 2. Periodic cell of the fractured medium.

when the medium and the excitation are homogenizable, i.e. when such an equivalent continuous macroscopic description exists.

On another hand, we are interested in deriving a continuous macroscopic model, equivalent to the finely heterogeneous porous media. This is only possible (Auriault, 1991a) under the condition that the macroscopic sample and the excitation acting on it are characterized by a length $l^{\prime \prime}$ very much larger than $l$ and $l^{\prime}$. This introduces a second small dimensionless parameter $l^{\prime} / l^{\prime \prime} \ll 1$.

In papers I and II, the quasi-static behaviour of such double porosity media with deformable matrices was investigated. The study was restricted to situations where the macroscopic description is a two-phase one. It was shown that depending on the relative value of the above small parameters, different macroscopic descriptions are obtained: classical single porosity Biot-like description, nonclassical description with a broken symmetry, non classical description with a broken symmetry and memory effects.

In this paper we aim at discovering the acoustic behaviour of double porosity saturated deformable media. Therefore, the characteristic length $l^{\prime \prime}$ of the excitation can be identified with the wavelength $\lambda$. By extending the results concerning the acoustics of single porosity media (Levy, 1979; Auriault, 1980, 1991b, 1992) we show that the two separations of scales introduced above impose that the behaviour of the pore fluid is necessarily quasi-static. Therefore, the first steps of the homogenization processes carried out in the papers I and II are still valid. The dynamics for the fracture fluid and the matrix has to be introduced at the end of the processes only. As a consequence, the results are a direct extension of the quasi-static ones and show the three different behaviours recalled above, but with additional dynamical terms and a generalized fracture seepage law.

The results of the papers I and II are recalled in Section 2, together with the main conclusions concerning the acoustics of a single porosity medium. Then Section 3 is devoted to the homogenization of the behaviour of a double porosity medium submitted to a dynamical excitation. A particular porous medium with a very simple geometry is investigated in Section 4. The medium is constituted of parallel slabs of an isotropic microporous media, separated by plane fractures. The simplicity of the 
geometry enables us to obtain analytical results for the effective coefficients. Finally, some remarks are given in Section 5 concerning the interpretation of acoustical laboratory experiments.

\section{Quasi-static Behaviour of a Double Porosity Medium and the Acoustics of Single Porosity Media}

We recall here the results of papers I and II for the quasi-static behaviour and the macroscopic description obtained by Levy (1979) and Auriault (1980), for the acoustics of a single porosity saturated and deformable medium. They will be useful in the next part for the analysis of the acoustics of the double porosity medium. In what follows the material constituting the matrix is assumed as elastic with small deformations and the fluid is viscous Newtonian and incompressible. The pores and the fractures are saturated. Let us recall firstly the single porosity description as introduced by Biot (1941) from a phenomenological approach:

$$
\begin{aligned}
& \nabla\left(\mathbf{c e}\left(\mathbf{u}_{s}\right)-\alpha p\right)=0, \\
& \nabla(\mathbf{K} \nabla p)=\alpha \frac{\partial\left(\mathbf{e}\left(\mathbf{u}_{s}\right)\right)}{\partial t}+\beta \frac{\partial p}{\partial t} .
\end{aligned}
$$

This system relates the pore pressure $p$ and the displacement $\mathbf{u}_{s}$ of the matrix. $\mathbf{c}, \alpha$ and $\beta$ are elastic tensors. $\mathbf{K}$ is the permeabilty and $\mathbf{e}$ is the deformation. This system exhibits a symmetry: the same tensor $\alpha$ appears in the two equations.

\subsection{QUASI-STATICS OF A DOUBLE POROSITY MEDIUM}

As pointed out above the macroscopic description depends on the relative value of the two parameters $l / l^{\prime}$ and $l^{\prime} / l^{\prime \prime}$. The different possibilities are shown in the Figure 3.

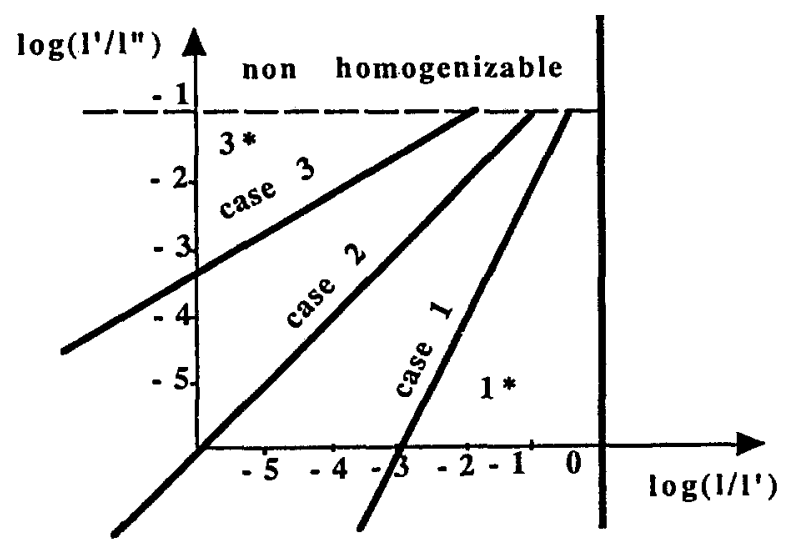

Fig. 3. Quasi-statics of a double porosity medium: homogenizable (case 1, 2 or 3) and nonhomogenizable situations, with respect to the two separations of scale. 
The homogenizability, i.e. the existence of a macroscopic description requires a small value of $l^{\prime} / l^{\prime \prime}$. It is generally considered that 0.1 represents the limit for the application of the homogenization process. This value is adopted in Figure 3. The description at the micro-scale is given by the following set of equations where the subscripts $s, p$ and $f$ stands for the solid, the fluid in the pores and the fluid in the fractures, respectively.

-- In the solid matrix the equations are the quasi-static momentum balance:

$$
\nabla \sigma_{s}=0,
$$

and the constitutive law (infinitesimal elasticity):

$$
\sigma_{\mathrm{s}}=\mathbf{a e}\left(\mathbf{u}_{s}\right)
$$

where the stress $\sigma_{s}$ is related to the small deformation

$$
\mathbf{e}\left(\mathbf{u}_{s}\right)=\frac{1}{2}\left(\nabla+{ }^{t} \nabla\right) \mathbf{u}_{s}
$$

by the elastic tensor $a$.

- In the fluid we have the quasi-static momentum balance:

$$
\mu \Delta \mathbf{v}_{\alpha}-\nabla p_{\alpha}=0, \quad \sigma_{\alpha}=-p_{\alpha} \mathbf{I}+2 \mu \mathbf{D},
$$

where $\mu$ is the viscosity, $\mathbf{v}$ the velocity, $p$ the pressure and $\mathbf{D}$ the rate of deformation: $\mathbf{D}=\mathbf{e}(\mathbf{v})$. The subscript $\alpha$ takes the values $p$ and $f$ in the pores and the fractures, respectively.

The incompressibility is written:

$$
\nabla \mathbf{v}_{\alpha}=0, \quad \alpha=p, f
$$

- The boundary conditions on $\Gamma$ are the continuity of the normal stresses and the velocities:

$$
\sigma_{\mathbf{s}} \mathbf{N}=\sigma_{p} \mathbf{N} \text { on } \Gamma \text {, }
$$

where $\mathbf{N}$ is the unit normal to $\Gamma$ and:

$$
\mathbf{v}_{s}=\mathbf{v}_{p}, \quad \text { on } \Gamma \text {. }
$$

- The boundary conditions on $\Gamma^{\prime}$ are the continuity of the normal stresses, the fluid pressure and the relative flux to the solid matrix.

The Equation (5) introduces the dimensionless numbers $Q_{\alpha}$ :

$$
Q_{\alpha}=\frac{\mu \Delta \mathbf{v}_{\alpha}}{\nabla p_{\alpha}}, \quad \alpha=p, f
$$

In our problem the driving force is a macroscopic pressure gradient:

$$
\nabla p_{\alpha}=O\left(\frac{p}{l^{\prime \prime}}\right)
$$


At the same time the velocity varies in the pores and the fractures, which gives for the viscous terms:

$$
\mu \Delta \mathbf{v}_{p}=O\left(\mu \frac{\mathbf{v}_{p}}{l^{2}}\right), \quad \mu \Delta \mathbf{v}_{f}=O\left(\mu \frac{\mathbf{v}_{f}}{l^{\prime 2}}\right)
$$

And from Equation (5) the viscous terms balance the pressure gradient:

$$
\frac{p}{l^{\prime \prime}}=O\left(\mu \frac{\mathbf{v}_{f}}{l^{\prime 2}}\right)=O\left(\mu \frac{\mathbf{v}_{p}}{l^{2}}\right) .
$$

Now, due to the very different sizes, the flow through the pores and the fractures introduces two characteristic times $T_{p}$ and $T_{f}$, respectively. We define them by:

$$
\mathbf{v}_{p}=\frac{\mathbf{u}_{p}}{T_{p}}, \quad \mathbf{v}_{f}=\frac{\mathbf{u}_{f}}{T_{f}},
$$

where $\mathbf{u}_{p}=\mathbf{u}_{f}$ is a reference displacement and where we assume $\mathbf{u}_{p}=\mathbf{u}_{f}=O\left(\mathbf{u}_{s}\right)$. Then, from Equation (9) we obtain:

$$
\frac{T_{\mathrm{f}}}{T_{p}}=O\left(\frac{l^{2}}{l^{2}}\right)
$$

Two cases exhibit nonclassical descriptions: case 1 where $l^{\prime} / l^{\prime \prime}=O\left(l^{2} / l^{\prime 2}\right)$ and case 2 where $l^{\prime} / l^{\prime \prime}=O\left(l / l^{\prime}\right)$. The other cases, $3,3^{*}, 1^{*}$ show classical descriptions.

(a) In case 1, the above dimensionless numbers are, when using $l^{\prime \prime}$ as the characteristic length of normalization:

$$
\begin{aligned}
& Q_{p l^{\prime \prime}}=\frac{\mu \Delta \mathbf{v}_{p}}{\nabla p_{p}}=O\left(\frac{\mu \mathbf{u}_{p} l^{2} T_{p}}{l^{\prime \prime 2} T_{f}^{\mu \mathbf{u}_{p}}}\right)=O\left(\frac{l^{2} T_{p}}{l^{\prime \prime 2} T_{f}}\right)=O\left(\varepsilon^{4}\right), \\
& Q_{f l^{\prime \prime}}=\frac{\mu \Delta \mathbf{v}_{\mathrm{f}}}{\nabla p_{\mathrm{f}}}=O\left(\frac{\mu \mathbf{u}_{\mathrm{f}} l^{\prime \prime}}{l^{\prime \prime 2} T_{f} p}\right)=O\left(\varepsilon^{4}\right), \\
& \frac{T_{f}}{T_{p}}=O\left(\frac{l^{2}}{l^{\prime 2}}\right)=O\left(\varepsilon^{2}\right), \varepsilon=\frac{l}{l^{\prime}} .
\end{aligned}
$$

At the first order of approximation, the corresponding macroscopic description is similar to the one given by M. A. Biot

$$
\begin{aligned}
& \nabla^{\prime \prime}\left(\mathbf{c}^{\prime \prime} \mathbf{e}^{\prime \prime}\left(\mathbf{u}_{s}\right)-\alpha^{\prime \prime} p^{0}\right)=0, \\
& \nabla^{\prime \prime}\left(\mathbf{K}_{f} \nabla^{\prime \prime} p^{0}\right)=\gamma^{\prime \prime} \frac{\partial \mathbf{e}^{\prime \prime}\left(\mathbf{u}_{s}^{0}\right)}{\partial t}+\beta^{\prime \prime} \frac{\partial p^{0}}{\partial t}
\end{aligned}
$$

but with a broken symmetry.

$$
\gamma^{\prime \prime} \neq \alpha^{\prime \prime}
$$


$\mathbf{c}^{\prime \prime}, \alpha^{\prime \prime}, \gamma^{\prime \prime}$ and $\beta^{\prime \prime}$ are here elastic coefficients. The macroscopic permeability $\mathbf{K}_{f}$ is related to the fractures, only.

(b) In case 2, the dimensionless numbers are

$$
\begin{aligned}
& Q_{p l^{\prime \prime}}=O\left(Q_{f l^{\prime \prime}}\right)=\frac{\mu \Delta \mathbf{v}_{\alpha}}{\nabla p_{\alpha}}=O\left(\frac{\mu \mathbf{u}_{p} l^{\prime \prime}}{l^{\prime \prime 2} T_{f} p}\right)=O\left(\frac{l^{2} T_{p}}{l^{\prime \prime 2} T_{f}}\right)=O\left(\varepsilon^{2}\right), \\
& \frac{T_{f}}{T_{p}}=O\left(\frac{l^{2}}{l^{\prime 2}}\right)=O\left(\varepsilon^{2}\right), \quad \varepsilon=\frac{l}{l^{\prime}} .
\end{aligned}
$$

When considering harmonic excitations of pulsation $\omega$, the macroscopic description is given by the Equations (13) and (14) which seem to be similar to the Equations (11) and (12)

$$
\begin{aligned}
& \nabla^{\prime \prime}\left(\mathbf{c}^{\prime \prime} \mathbf{e}^{\prime \prime}\left(\mathbf{u}_{s}^{0}\right)-\alpha^{\prime \prime} p_{f}^{0}\right)=0, \\
& \nabla^{\prime \prime}\left(\mathbf{K}_{f} \nabla^{\prime \prime} p_{f}^{0}\right)=\gamma^{\prime \prime} i \omega \mathbf{e}^{\prime \prime}\left(\mathbf{u}_{s}^{0}\right)+\beta^{\prime \prime} i \omega p_{f}^{0}, \quad \mathbf{K}_{f}=\left\langle\mathbf{K}_{f}\right\rangle_{\Omega^{\prime}},
\end{aligned}
$$

where $\mathbf{K}_{f}$ is again the permeability tensor of the fractures. But $\mathbf{c}^{\prime \prime}, \alpha^{\prime \prime}, \gamma^{\prime \prime}$ and $\beta^{\prime \prime}$ are now complex valued and $\omega$ dependent. As in case 1 , the symmetry is broken, $\gamma^{\prime \prime} \neq \alpha^{\prime \prime}$. Under transient excitations the medium will show memory effects. Concerning the pressures, the macroscopic description comprises the fracture pressure field only. But in opposition to case 1, the pore pressure is here different from the pressure in the fractures.

(c) Case $1^{*}$ is defined by

$$
\frac{l^{\prime}}{l^{\prime \prime}}=O\left(\frac{l^{q}}{l^{q}}\right), \quad q \geqslant 3 .
$$

In case $1^{*}, q=3$, we recover a classical, symmetrical BIOT's description, even if, due to the separation of scales $l / l^{\prime} \ll 1$, the macroscopic permeability is still the fracture permeability. If $q$ is increasing, the pore and fracture scales become nonseparated and the medium transforms to a single porous medium.

(d) Cases 3 and $3^{*}$ are characterized by

$$
\frac{l^{\prime q}}{l^{\prime \prime q}}=O\left(\frac{l}{l^{\prime}}\right), q \geqslant 2 .
$$

The macroscopic behaviour is also modelled by classical symmetrical BIOT's description. The fracture pressure field is required only. But in opposition to case 1, and as in case 2, the pore pressure is different from the pressure in the fractures. At the first-order approximation there is no relative movement of the pore fluid with respect to the matrix. The macroscopic behaviour of the matrix plus the pore fluid is that of a monophasic elastic medium. And the pore fluid does not contribute to the seepage. 


\subsection{ACOUSTICS OF A SINGLE POROSITY MEDIUM}

We recall the macroscopic two-phase behaviour of a saturated deformable medium. With a view to avoid confusion with the double porosity medium, the characteristic length of the pores is denoted here $l^{*}$. This $l^{*}$ will correspond to $l$ or $l^{\prime}$ of the double porosity medium, respectively. Again $l^{\prime \prime}$ is the characteristic macroscopic length. It can be identified to the wavelength. The solid and the fluid parts of the period are $\Omega_{s}$ are $\Omega_{F}$ respectively. $\Gamma^{*}$ is the interface.

The description at the pore level is given by the Navier equations in the solid part and the Navier-Stokes equation in the fluid part. Classically the interface conditions are the continuity of the displacement and the normal stress:

$$
\begin{aligned}
& \nabla \sigma_{s}=\rho_{s} \frac{\partial^{2} \mathbf{u}_{s}}{\partial t^{2}}, \\
& \sigma_{s}=\mathbf{a e}\left(\mathbf{u}_{s}\right) \text { in } \Omega_{s}, \\
& \nabla \sigma_{F}=\rho_{F}\left(\frac{\partial \mathbf{v}_{F}}{\partial t}+\left(\mathbf{v}_{F} \nabla\right) \mathbf{v}_{F}\right), \\
& \sigma_{F}=2 \mu \mathbf{D}-p \mathbf{l}, \\
& \nabla \mathbf{v}_{F}=0 \text { in } \Omega_{F} \\
& {[\sigma] \mathbf{N}=0, \text { on } \Gamma^{*},} \\
& {[\mathbf{u}]=0 ; \text { on } \Gamma^{*} .}
\end{aligned}
$$

We admit that the macroscopic stresses, the densities and the displacements are of the same order of magnitude

$$
\sigma_{s}=O\left(\sigma_{F}\right), \quad \mathbf{u}_{s}=O\left(\mathbf{u}_{F}\right), \quad \rho_{s}=O\left(\rho_{F}\right) .
$$

To be homogenizable the dynamical description of the solid requires

$$
P_{l^{\prime \prime}}=\frac{\rho_{s} \frac{\partial^{2} \mathbf{u}_{s}}{\partial t^{2}}}{\nabla \sigma_{s}}=O(1),
$$

where, as above, the index $l^{\prime \prime}$ shows the characteristic length used to calculate the dimensionless number $P$.

Due to the small amplitude of the vibration, the Reynolds number is negligible and from now we disregard the convective inertial term of the Navier-Stokes equation.

Since we focus on the two-phase macroscopic description, the number $Q$ has the same order of magnitude as $Q_{f}$ :

$$
Q_{l^{\prime \prime}}=\frac{\mu \Delta \mathbf{v}_{F}}{\nabla p}=O\left(\varepsilon^{2}\right)
$$


The acoustics of the fluid imposes a ratio of the inertial term to the pressure term being of the order of magnitude 1 at the macroscopic level:

$$
T_{l^{\prime \prime}}=\frac{\rho_{f} \frac{\partial \mathbf{v}_{F}}{\partial t}}{\nabla p}=O(1) .
$$

These values of the dimensionless numbers imply the following order of magnitude of the transient Reynolds number

$$
R t_{l^{\prime \prime}}=\frac{\rho_{f} \frac{\partial \mathbf{v}_{F}}{\partial t}}{\mu \Delta \mathbf{v}_{F}}=O\left(\varepsilon^{-2}\right)
$$

that corresponds to a local transient Reynolds number in the form

$$
R t_{l^{*}}=O(1)
$$

Higher values of $R_{t}$ lead to nonhomogenizable situations.

The macroscopic description at a constant pulsation $\omega$ comprises two balance equations. The first one is a volume balance coupled with a generalized seepage law:

$$
\begin{aligned}
& \nabla\left(\left\langle\mathbf{v}_{F}\right\rangle-n i \omega \mathbf{u}_{s}\right)=-i \omega \alpha \mathbf{e}\left(\mathbf{u}_{s}\right)-i \omega \beta p, \\
& \left\langle\mathbf{v}_{F}\right\rangle-n i \omega \mathbf{u}_{s}=-\mathbf{K}\left(\nabla p-\omega^{2} \rho_{F} \mathbf{u}_{s}\right) .
\end{aligned}
$$

The second one is a momentum balance for the bulk medium

$$
\begin{aligned}
& \nabla\left\langle\sigma^{T}\right\rangle=-\left\langle\rho_{s}\right\rangle \omega^{2} \mathbf{u}_{s}+i \rho_{F} \omega \mathbf{v}_{F}, \\
& \left\langle\sigma^{T}\right\rangle=\mathbf{c e}\left(\mathbf{u}_{s}\right)-\alpha p .
\end{aligned}
$$

Here, $\sigma^{T}$ is the total stress defined by

$$
\sigma^{T}=\left\{\begin{array}{ll}
\sigma_{s} & \text { in } \Omega_{s} \\
\sigma_{F} & \text { in } \Omega_{F}
\end{array} .\right.
$$

This description can be shown (Auriault, 1981) to be equivalent to the one of Biot (1956). The tensor $K$ is a generalized permeability tensor which is $\omega$ depending and complex valued. The tensors $\mathbf{c}, \alpha$ an $\beta$ are the quasi-static elastic tensors, already introduced. We note the symmetry introduced by the same tensor $\alpha$ appearing in Equations (22) and (23).

\section{Acoustics of a Double Porosity Medium}

The local description is the same as in the quasi-statics case, with additional inertial terms:

$$
\nabla \sigma_{s}=\rho_{s} \frac{\partial^{2} \mathbf{u}_{s}}{\partial t^{2}}
$$




$$
\begin{aligned}
& \sigma_{s}=\mathbf{a e}\left(\mathbf{u}_{s}\right), \quad \text { in } \Omega_{\mathrm{s}}, \\
& \nabla \sigma_{\alpha}=\rho_{F}\left(\frac{\partial \mathbf{v}_{\alpha}}{\partial t}+\left(\mathbf{v}_{\alpha} \nabla\right) \mathbf{v}_{\alpha}\right), \quad \alpha=p, f, \\
& \sigma_{\alpha}=2 \mu \mathbf{D}\left(\mathbf{v}_{\alpha}\right)-p_{\alpha} \mathbf{l}, \\
& \nabla \mathbf{v}_{\alpha}=0, \quad \alpha=p, f, \quad \text { in } \Omega_{\alpha}
\end{aligned}
$$

The boundary conditions on $\Gamma$ are the continuity of the normal stresses and the velocities:

$$
\sigma_{s} \mathbf{N}=\sigma_{p} \mathbf{N}, \quad \text { on } \Gamma,
$$

where $\mathbf{N}$ is the unit normal to $\Gamma$ and:

$$
\mathbf{v}_{s}=\mathbf{v}_{p}, \quad \text { on } \Gamma \text {. }
$$

The boundary conditions on $\Gamma^{\prime}$ are the continuity of the normal stress, the fluid pressure and the flux relative to the solid matrix.

Firstly, we focus on the acoustics corresponding to case 2 . Therefore, the dimensionless numbers $Q_{\alpha}, \alpha=p, f$, and the ratio of the two characteristic times take the following values, already introduced in the quasi-static case;

$$
Q_{p l^{\prime \prime}}=O\left(Q_{f l^{\prime \prime}}\right)=\frac{\mu \Delta \mathbf{v}_{\alpha}}{\nabla p_{\alpha}}=O\left(\varepsilon^{2}\right), \quad \frac{T_{f}}{T_{p}}=O\left(\frac{l^{2}}{l^{\prime 2}}\right)=O\left(\varepsilon^{2}\right) .
$$

On the other hand, homogenizable dynamics of the solid part implies (Auriault, 1991):

$$
P_{l^{\prime \prime}}=\frac{\rho_{s} \frac{\partial^{2} \mathbf{u}_{s}}{\partial t^{2}}}{\nabla \sigma_{s}}=O(1)
$$

and homogenizable dynamics of the fluid part imposes the local transient Reynolds numbers to be equal or less than $O(1)$. Since they take the value

$$
R_{t p l}=\frac{\rho \omega l^{2}}{\mu}, \quad R_{t f l^{\prime}}=\frac{\rho \omega l^{2}}{\mu},
$$

the ratio between the two is given by

$$
\frac{R_{t p l}}{R_{t f l^{\prime}}}=\frac{l^{2}}{l^{2}}=O\left(\varepsilon^{2}\right)
$$

Therefore, the only possibility for a dynamical behaviour to occur is

$$
R_{t p l}=O\left(\varepsilon^{2}\right), \quad R_{t f l^{\prime}}=O(1),
$$

which corresponds to

$$
R_{t p l^{\prime \prime}}=R_{t f l^{\prime \prime}}=O\left(\varepsilon^{-2}\right) .
$$


With the above estimations, the dimensionless form of the local description takes the following form:

$$
\begin{aligned}
& \nabla \sigma_{s}=\rho_{s} \frac{\partial^{2} \mathbf{u}_{s}}{\partial t^{2}}, \\
& \sigma_{s}=\mathbf{a e}\left(\mathbf{u}_{s}\right), \quad \text { in } \Omega_{s}, \\
& \nabla \sigma_{\alpha}=\rho_{F} \frac{\partial \mathbf{v}_{\alpha}}{\partial t}, \quad \alpha=p, f, \\
& \sigma_{\alpha}=2 \mu \varepsilon^{2} \mathbf{D}\left(\mathbf{v}_{\alpha}\right)-p_{\alpha} l, \\
& \nabla \mathbf{v}_{\alpha}=0, \quad \alpha=p, f \text { in } \Omega \alpha, \\
& \sigma_{s} \mathbf{N}=\sigma_{p} \mathbf{N}, \\
& \mathbf{u}_{s}=\mathbf{u}_{p} \quad \text { on } \Gamma, \\
& p_{p}=p_{f}, \\
& \left(\left\langle\sigma_{s}\right\rangle_{\Omega}+\left\langle\sigma_{p}\right\rangle_{\Omega}\right) \mathbf{N}^{\prime}=\sigma_{f} \mathbf{N}^{\prime}
\end{aligned}
$$

and

$$
\mathbf{v}_{f}-\left\langle\mathbf{v}_{s}\right\rangle_{\Omega}=\left\langle\mathbf{v}_{p}\right\rangle_{\Omega} \text { on } \Gamma^{\prime} .
$$

The homogenization process is carried out in the same way as in paper II. The three characteristic lengths introduce three space variables $\mathbf{x}, \mathbf{x}^{\prime}$ and $\mathbf{x}^{\prime \prime}$, corresponding respectively to the pore, the fracture and the macroscopic scales. They are related to each other by the powers of $\varepsilon$ :

$$
\mathbf{x}=\varepsilon^{-2} \mathbf{x}^{\prime \prime}, \quad \mathbf{x}^{\prime}=\varepsilon^{-1} \mathbf{x}^{\prime \prime} .
$$

In the same way, the two characteristic times $T_{p}$ and $T_{f}$ for the flows through the pores and the fractures, respectively, introduce two time variables $t$ and $T$, with $T=\varepsilon^{2} t$.

As a consequence, by following the procedure precised in paper I, each unknown $\Phi$ will be looked for in the form:

$$
\Phi=\Phi^{0}\left(\mathbf{x}, \mathbf{x}^{\prime}, \mathbf{x}^{\prime \prime}, t, T\right)+\varepsilon \Phi^{1}\left(\mathbf{x}, \mathbf{x}^{\prime}, \mathbf{x}^{\prime \prime}, t, T\right)+\cdots,
$$

where

$$
\mathbf{x}=\varepsilon^{-2} \mathbf{x}^{\prime \prime}, \quad \mathbf{x}^{\prime}=\varepsilon^{-1} \mathbf{x}^{\prime \prime}, \quad T=\varepsilon^{2} t,
$$

with $\Phi^{i} \Omega$ and $\Omega^{\prime}$ periodic. The expression (41) means that $\mathbf{x}^{\prime \prime}$ and $t$ were chosen as driving space and time variable, respectively. It is coherent with our choice of $l^{\prime \prime}$ and $T_{f}$ to make the local description dimensionless. The homogenization process consists in introducing such asymptotic developments into the dimensionless local description and in collecting like powers of $\varepsilon$. When comparing with the quasi-static case, Annexe A of paper II, it is clear that the new inertial terms intervene in the equations 
at the zero order of magnitude only. Therefore, the process carried out in paper II is valid until this order. It is easy to check that the bulk momentum balance at constant pulsation in Equation (13) is modified by simply adding the bulk inertial term:

$$
\begin{aligned}
\nabla^{\prime \prime}\left(\mathbf{c}^{\prime \prime} \mathbf{e}^{\prime \prime}\left(\mathbf{u}_{s}^{0}\right)-\alpha^{\prime \prime} p_{f}^{0}\right)= & -\left((l-n) \rho_{s}+n \rho_{F}\right)\left(l-n^{\prime}\right) \omega^{2} \mathbf{u}_{s}^{0}+ \\
& +i \omega \rho_{F}\left\langle\mathbf{v}_{f}^{0}\right\rangle .
\end{aligned}
$$

Concerning the fluid part, the modification concerns the set of equations giving the local velocity field in the fractures:

$$
\begin{aligned}
& \nabla^{\prime} \sigma_{f}^{1}+\nabla^{\prime \prime} \sigma_{f}^{0}=i \omega \rho_{F} \mathbf{v}_{f}^{0}, \quad \sigma_{f}^{1}=-p_{f}^{1} \mathbf{I}+2 \mu \mathbf{e}^{\prime}\left(\mathbf{v}_{f}^{0}\right), \\
& \mathbf{v}_{f}^{0}=\mathbf{v}_{s}^{0}
\end{aligned}
$$

on $\Gamma^{\prime}$. Therefore, we have

$$
\mathbf{v}_{f}^{0}-\mathbf{v}_{s}^{0}=-\mathbf{k}_{f}\left(\mathbf{x}^{\prime}\right)\left(\nabla^{\prime \prime} p_{f}^{0}-\omega^{2} \rho_{F} \mathbf{u}_{s}^{0}\right),
$$

where $\mathbf{k}_{f}$ is now $\omega$ depending and complex valued. The Darcy law is replaced by a generalized seepage law of the same type as in Section 2.2:

$$
\left\langle\mathbf{v}_{f}^{0}\right\rangle-n^{\prime} \mathbf{v}_{s}^{0}=-\mathbf{K}_{f}\left(\nabla^{\prime \prime} p_{f}^{0}-\omega^{2} \rho_{F} \mathbf{u}_{s}^{0}\right), \quad \mathbf{K}_{f}=\left\langle\mathbf{k}_{f}\right\rangle .
$$

The volume balance rests unchanged:

$$
\nabla^{\prime \prime}\left(\left\langle\mathbf{v}_{f}^{0}\right\rangle-n^{\prime} i \omega \mathbf{u}_{s}^{0}\right)=-\gamma^{\prime \prime} i \omega \mathbf{e}^{\prime \prime}\left(\mathbf{u}_{s}^{0}\right)-\beta^{\prime \prime} i \omega p_{f}^{0} .
$$

Equations (42)-(44) represent the macroscopic description of the acoustics of the double porosity medium. The effective tensors $\mathbf{c}^{\prime \prime}, \alpha^{\prime \prime}, \gamma^{\prime \prime}$, and $\beta^{\prime \prime}$ are those of the quasi-static behaviour. They are $\omega$ depending and complex valued and show viscoelastic-like memory effects. The acoustics description is obtained from the quasi-static description by adding inertial terms and replacing the classical permeability tensor by the generalized permeability tensor which is also $\omega$ depending and complex valued.

The investigation of case 1 leads to the same conclusion for the acoustics. The description is shown to be (42)-(44), but where the effective tensors $\mathbf{c}^{\prime \prime}, \alpha^{\prime \prime}, \gamma^{\prime \prime}$ and $\beta^{\prime \prime}$ are now elastic tensors (i.e. real and $\omega$ independent tensors).

\section{Double Porosity Medium: an Example}

We investigate the macroscopic behaviour of a particular double porosity medium composed of parallel micro-porous slabs separated from each others by flat fractures (see Figure 4 for the notations). The space variables $x^{\prime \prime}, y^{\prime \prime}, z^{\prime \prime}$ are for the macroscopic description, whereas $x^{\prime}, y^{\prime}$ and $z^{\prime}$ stands for the space variables at the fracture level. The slabs and the fractures are parallel to the $x^{\prime \prime} z^{\prime \prime}$ (or the $x^{\prime} z^{\prime}$ ) plane and they are perpendicular to the $y^{\prime \prime}$ (or the $y^{\prime}$ ) axis. The medium is periodic with a period $l^{\prime}$ in the $y^{\prime}$ direction. Obviously, the periodicity is 


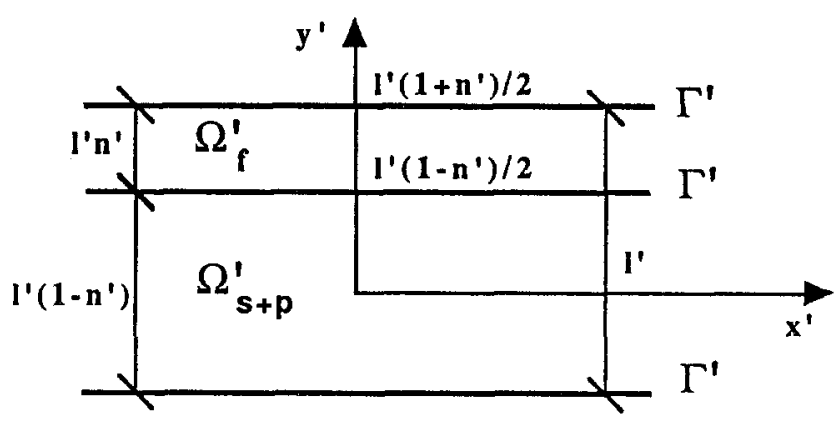

Fig. 4. Layered microporous fractured medium.

arbitrary in the $x^{\prime}$ and $z^{\prime}$ directions. The behaviour of the micro-porous is supposed to be known and we aim at discovering the effective coefficients for plane dilational waves propagating in the parallel direction to the slabs and fractures, i.e. in the $x^{\prime \prime}$ direction.

The first local problem to be solved is given by the set (A.21b, A.23, A.24, A.15c, A.14a) of paper II:

$$
\begin{aligned}
& \nabla^{\prime}\left(\left\langle\mathbf{k}_{p}\right\rangle_{\Omega} \nabla^{\prime} p_{p}^{0}\right)=\alpha^{\prime} i \omega\left(\mathbf{e}^{\prime}\left(\mathbf{u}_{s}^{l}\right)+\mathbf{e}^{\prime \prime}\left(\mathbf{u}_{s}^{0}\right)\right)+\beta^{\prime} i \omega p_{p}^{0}, \\
& \nabla^{\prime \prime}\left(\left\langle\sigma_{s}^{0}\right\rangle_{\Omega}-n p_{p}^{0} \mathbf{I}\right)=0, \\
& \left\langle\sigma_{s}^{0}\right\rangle_{\Omega}-n p_{p}^{0} \mathbf{I}=\mathbf{c}^{\prime}\left(\mathbf{e}^{\prime}\left(\mathbf{u}_{s}^{l}\right)+\mathbf{e}^{\prime \prime}\left(\mathbf{u}_{s}^{0}\right)\right)-\alpha^{\prime} p_{p}^{0}
\end{aligned}
$$

in the micro-porous medium and:

$$
\begin{aligned}
& \left(\left\langle\sigma_{s}^{0}\right\rangle_{\Omega}-n p_{p}^{0} \mathbf{I}\right) \mathbf{N}^{\prime}=-p_{f}^{0} \mathbf{N}^{\prime}, \\
& p_{p}^{0}=p_{f}^{0}\left(\mathbf{x}^{\prime \prime}\right), \quad \text { on } \Gamma^{\prime} .
\end{aligned}
$$

This set represents a boundary-value problem for $\mathbf{u}_{s}^{l}$ and $p_{p}^{0}$ under harmonic excitation. They where shown to be linear functions of $\mathbf{e}^{\prime \prime}\left(\mathbf{u}_{s}^{0}\right)$ and $p_{f}^{0}$ which depend on the variable $x^{\prime \prime}$, only:

$$
\begin{aligned}
& \mathbf{u}_{\mathrm{s}}^{l}=\xi^{\prime}\left(\mathbf{x}^{\prime}\right) \mathbf{e}^{\prime \prime}\left(\mathbf{u}_{s}^{0}\right)-\eta^{\prime}\left(\mathbf{x}^{\prime}\right) p_{f}^{0}+\hat{\mathbf{u}}_{s}^{l}\left(\mathbf{x}^{\prime \prime}, t, T\right), \\
& p_{p}^{0}=v^{\prime}\left(\mathbf{x}^{\prime}\right) \mathbf{e}^{\prime \prime}\left(\mathbf{u}_{s}^{0}\right)+\tau^{\prime}\left(\mathbf{x}^{\prime}\right) p_{f}^{0} .
\end{aligned}
$$

It is important to notice again that the tensors $\xi^{\prime}, \eta^{\prime}, v^{\prime}$ and the scalar $\tau^{\prime}$ are here complex valued and $\omega$ dependent.

For the sake of simplicity we limit ourselves to an isotropic and homogeneous micro-porous medium with a matrix made of an incompressible elastic material. Therefore, $\alpha^{\prime}=\mathbf{I}, \beta^{\prime}=0$ and $\left\langle\mathbf{k}_{p}\right\rangle_{\Omega}=\mathbf{K}_{p} \mathbf{I}$ and we note $\lambda^{\prime}$ and $\mu^{\prime}$ the Lamé coefficients of the matrix.

On the other hand, due to the arbitrary periodicity in the $x^{\prime}$ and $y^{\prime}$ directions, the unknowns are $x^{\prime}$ and $y^{\prime}$ independent. Denoting $u^{1}, v^{1}$, and $w^{1}$ the components of $\mathbf{u}_{s}^{l}$, the corresponding deformation is given by 


$$
\mathbf{e}^{\prime}\left(\mathbf{u}_{s}^{1}\right)=\left(\begin{array}{ccc}
0 & \frac{1}{2} \frac{\mathrm{d} u^{1}}{\mathrm{~d} y^{\prime}} & 0 \\
\frac{1}{2} \frac{\mathrm{d} u^{1}}{\mathrm{~d} y^{\prime}} & \frac{\mathrm{d} v^{1}}{\mathrm{~d} y^{\prime}} & \frac{1}{2} \frac{\mathrm{d} w^{1}}{\mathrm{~d} y^{\prime}} \\
0 & \frac{1}{2} \frac{\mathrm{d} w^{1}}{\mathrm{~d} y^{\prime}} & 0
\end{array}\right)
$$

Since we limit ourselves to plane dilatational waves propagating in the $x^{\prime \prime}$ direction, we have $\mathbf{u}_{s}^{0}=\left(u^{0}, 0,0\right)$ and

$$
\mathbf{e}^{\prime \prime}\left(\mathbf{u}_{s}^{0}\right)=\left(\begin{array}{ccc}
\frac{\mathrm{d} u^{0}}{\mathrm{~d} x^{\prime \prime}} & 0 & 0 \\
0 & 0 & 0 \\
0 & 0 & 0
\end{array}\right) .
$$

With all this simplifications we are left with the following set of differential equations

$$
\begin{aligned}
& \mathbf{K}_{p} \frac{\mathrm{d}^{2} p_{p}^{0}}{\mathrm{~d} y^{\prime 2}}=i \omega\left(\frac{\mathrm{d} y^{1}}{\mathrm{~d} y^{\prime}}+\frac{\mathrm{d} u^{0}}{\mathrm{~d} x^{\prime \prime}}\right), \quad \frac{\mathrm{d}}{\mathrm{d} y^{\prime}}\left(\mu^{\prime} \frac{\mathrm{d} u^{1}}{\mathrm{~d} y^{\prime}}\right)=0, \\
& \frac{\mathrm{d}}{\mathrm{d} y^{\prime}}\left(\lambda^{\prime}\left(\frac{\mathrm{d} v^{1}}{\mathrm{~d} y^{\prime}}+\frac{\mathrm{d} u^{0}}{\mathrm{~d} x^{\prime \prime}}\right)+2 \mu^{\prime} \frac{\mathrm{d} v^{1}}{\mathrm{~d} y^{\prime}}-p_{p}^{0}\right)=0, \\
& \frac{\mathrm{d}}{\mathrm{d} y^{\prime}}\left(\mu^{\prime} \frac{\mathrm{d} w^{1}}{\mathrm{~d} y^{\prime}}\right)=0,
\end{aligned}
$$

with on $\Gamma^{\prime}$ :

$$
\begin{array}{ll}
\mu^{\prime} \frac{\mathrm{d} u^{1}}{\mathrm{~d} y^{\prime}}=0, & \lambda^{\prime}\left(\frac{\mathrm{d} v^{l}}{\mathrm{~d} y^{\prime}}+\frac{\mathrm{d} u^{0}}{\mathrm{~d} x^{\prime \prime}}\right)+2 \mu^{\prime} \frac{\mathrm{d} v^{1}}{\mathrm{~d} y^{\prime}}-p_{p}^{0}=-p_{f}^{0}, \\
\mu^{\prime} \frac{\mathrm{d} w^{1}}{\mathrm{~d} y^{\prime}}=0, & p_{p}^{0}=p_{f}^{0} .
\end{array}
$$

The solution is easy to obtain. For $p_{p}^{0}$ it becomes

$$
p_{p}^{0}=v^{\prime} \frac{\mathrm{d} u^{0}}{\mathrm{~d} x^{\prime \prime}}+\tau^{\prime} p_{f}^{0}
$$

with $\tau^{\prime}=1$ and

$$
v^{\prime}=2 \mu^{\prime}\left(\frac{\cos \left(\mathrm{y}^{\prime}\left(\frac{-i \omega}{K_{\mathrm{p}}\left(\lambda^{\prime}+2 \mu^{\prime}\right)}\right)^{1 / 2}\right)}{\cos \left(\frac{l^{\prime}\left(1-n^{\prime}\right)}{2}\left(\frac{-i \omega}{K_{\mathrm{p}}\left(\lambda^{\prime}+2 \mu^{\prime}\right)}\right)^{1 / 2}\right)}-1\right) .
$$


To determine the displacement we need only the spatial derivatives

$$
\frac{\mathrm{d} u^{1}}{\mathrm{~d} y^{\prime}}=0, \quad \frac{\mathrm{d} v^{1}}{\mathrm{~d} y^{\prime}}=\frac{1}{\lambda^{\prime}+2 \mu^{\prime}}\left(p_{p}^{0}-p_{f}^{0}-\lambda^{\prime} \frac{\mathrm{d} u^{0}}{\mathrm{~d} x^{\prime \prime}}\right), \quad \frac{\mathrm{d} w^{1}}{\mathrm{~d} y^{\prime}}=0 .
$$

The total macroscopic stress is given by:

$$
\begin{aligned}
\left\langle\left\langle\sigma_{T 11}^{0}\right\rangle_{\Omega \Omega^{\prime}}\right. & =\left\langle\lambda^{\prime}\left(\frac{\mathrm{d} v^{1}}{\mathrm{~d} y^{\prime}}+\frac{\mathrm{d} u^{0}}{\mathrm{~d} x^{\prime \prime}}\right)+2 \mu^{\prime} \frac{\mathrm{d} u^{0}}{\mathrm{~d} x^{\prime \prime}}-p_{p}^{0}\right\rangle_{\Omega^{\prime}}-n^{\prime} p_{f}^{0} \\
& =\mathbf{c}_{1}^{\prime \prime} \frac{\mathrm{d} u^{0}}{\mathrm{~d} x^{\prime \prime}}-\alpha_{1}^{\prime \prime} p_{p}^{0}
\end{aligned}
$$

with $\alpha_{1}^{\prime \prime}=1$ and:

$$
\begin{aligned}
& \mathbf{c}_{1}^{\prime \prime}=4 \mu^{\prime}\left(1-n^{\prime}\right)\left[1-\frac{2 \mu^{\prime}}{\left(1-n^{\prime}\right) l^{\prime}\left(\lambda^{\prime}+2 \mu^{\prime}\right)} \frac{\tan \left(\frac{l^{\prime}\left(1-n^{\prime}\right)}{2}\left(\frac{-i \omega}{K_{\mathrm{p}}\left(\lambda^{\prime}+2 \mu^{\prime}\right)}\right)^{1 / 2}\right)}{\left(\frac{-i \omega}{K_{\mathrm{p}}\left(\lambda^{\prime}+2 \mu^{\prime}\right)}\right)^{1 / 2}}\right] \\
& \left\langle\left\langle\sigma_{T 22}^{0}\right\rangle_{\Omega \Omega^{\prime}}=\left\langle\lambda^{\prime}\left(\frac{\mathrm{d} v^{1}}{\mathrm{~d} y^{\prime}}+\frac{\mathrm{d} u^{0}}{\mathrm{~d} x^{\prime \prime}}\right)+2 \mu^{\prime} \frac{\mathrm{d} v^{1}}{\mathrm{~d} y^{\prime}}-p_{p}^{0}\right\rangle_{\Omega^{\prime}}-n^{\prime} p_{f}^{0}=-p_{f}^{0},\right. \\
& \left\langle\left\langle\sigma_{T 33}^{0}\right\rangle_{\Omega \Omega^{\prime}}=\left\langle\lambda^{\prime}\left(\frac{\mathrm{d} v^{1}}{\mathrm{~d} y^{\prime}}+\frac{\mathrm{d} u^{0}}{\mathrm{~d} x^{\prime \prime}}\right)-p_{p}^{0}\right\rangle_{\Omega^{\prime}}-n^{\prime} p_{f}^{0}=\mathbf{c}_{3}^{\prime \prime} \frac{\mathrm{d} u^{0}}{\mathrm{~d} x^{\prime \prime}}-\alpha_{3}^{\prime \prime} p_{p}^{0}\right.
\end{aligned}
$$

with $\alpha_{3}^{\prime \prime}=1$ and

$$
\begin{aligned}
& \mathbf{c}_{3}^{\prime \prime}=2 \mu^{\prime}\left(1-n^{\prime}\right)\left[1-\frac{4 \mu^{\prime}}{\left(1-n^{\prime}\right) l^{\prime}\left(\lambda^{\prime}+2 \mu^{\prime}\right)} \frac{\tan \left(\frac{l^{\prime}\left(1-n^{\prime}\right)}{2}\left(\frac{-i \omega}{K_{\mathrm{p}}\left(\lambda^{\prime}+2 \mu^{\prime}\right)}\right)^{1 / 2}\right)}{\left(\frac{-i \omega}{K_{\mathrm{p}}\left(\lambda^{\prime}+2 \mu^{\prime}\right)}\right)^{1 / 2}}\right], \\
& \left\langle\left\langle\sigma_{T 12}^{0}\right\rangle_{\Omega \Omega^{\prime}}=\left\langle\mu^{\prime} \frac{\mathrm{d} u^{1}}{\mathrm{~d} y^{\prime}}\right\rangle_{\Omega^{\prime}}=0, \quad\left\langle\left\langle\sigma_{T 13}^{0}\right\rangle_{\Omega \Omega^{\prime}}=0,\right.\right. \\
& \left\langle\left\langle\sigma_{T 23}^{0}\right\rangle\right\rangle_{\Omega \Omega^{\prime}}=\left\langle\mu^{\prime} \frac{\mathrm{d} w^{1}}{\mathrm{~d} y^{\prime}}\right\rangle_{\Omega^{\prime}}=0 .
\end{aligned}
$$

Finally the bulk momentum balance of interest, in the $x^{\prime \prime}$ direction, is given by

$$
\begin{aligned}
& \frac{\mathrm{d}}{\mathrm{d} x^{\prime \prime}}\left(c_{1}^{\prime \prime} \frac{\mathrm{d} u^{0}}{\mathrm{~d} x^{\prime \prime}}-p_{f}^{0}\right)= \\
& \quad-\left((1-n) \rho_{\mathrm{s}}+n \rho_{F}\right)\left(1-n^{\prime}\right) \omega^{2} u^{0}+i \omega \rho_{F}\left\langle\mathbf{v}_{f}^{0}\right\rangle
\end{aligned}
$$


Decomposing the viscoelastic coefficient $c_{1}^{\prime \prime}$ into real and imaginary parts

$$
\mathbf{c}_{1}^{\prime \prime}=\mathbf{c}_{r}^{\prime \prime}+i \mathbf{c}_{i}^{\prime \prime},
$$

we introduce the quality factor $Q_{a}$ of the saturated microporous medium in the form

$$
Q_{a}=\frac{\mathbf{c}_{r}^{\prime \prime}}{\mathbf{c}_{i}^{\prime \prime}}
$$

The inverse of the quality factor $Q_{a}$ is shown on Figure 5 with respect to the dimensionless pulsation $\omega_{a}$

$$
\omega_{a}=\frac{\omega l^{\prime 2}\left(1-n^{\prime}\right)^{2}}{2 \mathrm{~K}_{\mathrm{p}}\left(\lambda^{\prime}+2 \mu^{\prime}\right)}=O\left(\frac{l^{\prime}}{\lambda_{P_{2}}}\right)^{2}
$$

where $\lambda_{P_{2}}$ is the wavelength of the $P_{2}$ wave of the saturated microporous medium at low frequency. The micro-porous matrix has the following characteristic corresponding to a Berea sandstone:

$$
\frac{\left(\lambda^{\prime}+2 \mu^{\prime}\right)}{\mu^{\prime}}=2.86 \text {. }
$$

The second problem to be solved concerns the flow in the fractures. It is given by the set

$$
\begin{aligned}
& \nabla^{\prime \prime} \sigma_{f}^{l}+\nabla^{\prime \prime}\left(p_{f}^{0} \mathrm{I}\right)=i \omega \rho_{F} \mathbf{v}_{f}^{0}, \quad \sigma_{f}^{l}=-p_{f}^{l} \mathrm{I}+2 \mu \mathbf{e}^{\prime}\left(\mathbf{v}_{f}^{0}\right), \\
& \mathbf{v}_{f}^{0}=\mathbf{v}_{s}^{0} \text { on } \Gamma^{\prime} .
\end{aligned}
$$

For flat fractures the solution is well known (see, for example, Borne, 1983). One obtains a generalized seepage law in the $x^{\prime \prime}$ direction:

$$
\left\langle\mathbf{v}_{f}^{0}\right\rangle-i \omega n^{\prime} u^{0}=-\mathbf{K}_{\mathrm{f}}\left(\frac{\mathrm{d} p_{f}^{0}}{\mathrm{~d} x^{\prime \prime}}-\omega^{2} \rho_{F} u^{0}\right)
$$

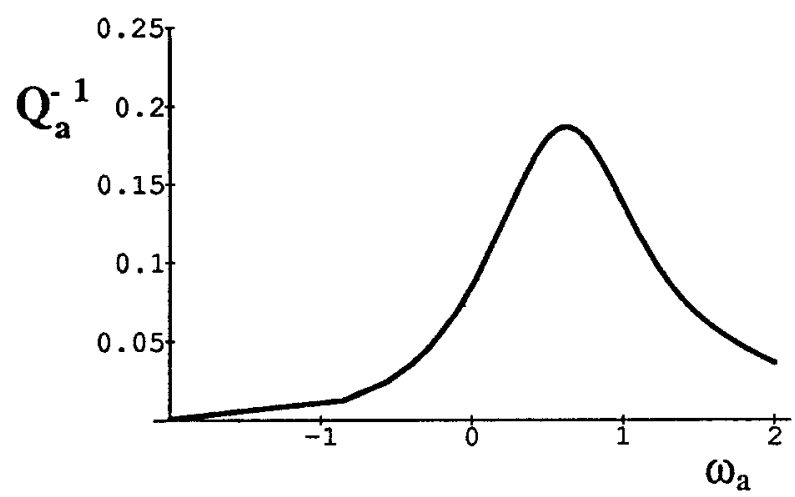

Fig. 5. Quality factor of the microporous subspace of the layered microporous fractured medium. 
with the acoustic permeability tensor:

$$
\mathbf{K}_{\mathrm{f}}=\frac{n^{\prime}}{i \omega \rho_{F}}\left[1-\frac{1}{l^{\prime} n^{\prime}}\left(\frac{\mu}{i \omega \rho_{F}}\right)^{1 / 2} \tanh \left(\left(\frac{i \omega \rho_{\mathrm{F}}}{\mu}\right)^{1 / 2} l^{\prime} n^{\prime}\right)\right] .
$$

The volume balance becomes:

$$
\frac{\mathrm{d}}{\mathrm{d} x^{\prime \prime}}\left(\left\langle v_{f}^{0}\right\rangle-n^{\prime} i \omega u^{0}\right)=-\gamma^{\prime \prime} i \omega \frac{\mathrm{d} u^{0}}{\mathrm{~d} x^{\prime \prime}}-\beta^{\prime \prime} i \omega p_{f}^{0} .
$$

with $\beta^{\prime \prime}=0$ and:

$$
\gamma^{\prime \prime}=1-\frac{4 \mu^{\prime}}{l^{\prime}\left(\lambda^{\prime}+2 \mu^{\prime}\right)} \frac{\tan \left(\frac{l^{\prime}\left(1-n^{\prime}\right)}{2}\left(\frac{-i \omega}{K_{\mathrm{p}}\left(\hat{\lambda}^{\prime}+2 \mu^{\prime}\right)}\right)^{1 / 2}\right)}{\left(\frac{-\mathrm{i} \omega}{K_{\mathrm{p}}\left(\lambda^{\prime}+2 \mu^{\prime}\right)}\right)^{1 / 2}} .
$$

We note again the broken symmetry:

$$
\gamma^{\prime \prime} \neq \alpha_{1}^{\prime \prime}=1
$$

Finally, the macroscopic description of dilatational plane wave propagating in the $x^{\prime \prime}$ direction is given by the Equations (48)-(50).

\section{Concluding Remarks}

The macroscopic behaviour of the double porosity medium shows two characteristic pulsations. The first one corresponds to the quasi-static viscous fluid flow through the micro-porous medium and is obtained from the Equations (45)-(47):

$$
\omega_{\mathrm{pv}}=O\left(\frac{\left\langle k_{\mathrm{p}}\right\rangle_{\Omega} c^{\prime}}{\alpha^{\prime} l^{\prime 2}}\right)
$$

The second one is the characteristic pulsation of the dynamical fluid flow in the fractures. It is obtained from the value of the local transient Reynolds of the fractures:

$$
\omega_{f d}=O\left(\frac{\mu}{\rho_{F} l^{\prime 2}}\right) .
$$

It is easy to check that for the most part of porous media:

$$
\omega_{f d} \ll \omega_{p v}
$$

The attenuation of double porosity effects will present two very well separated peaks at these two characteristic frequencies. The first one, at low frequency, is the dissipation peak of the fracture flow. The second one, at higher frequency, is the dissipative peak corresponding to the resonance of the diffusive $P_{2}$ wave of 
the saturated microporous medium excitated by the fracture flow. On the other hand we see that the pore fluid cannot be dynamically excitated if we restrict ourselves to homogenizable situations. The characteristic pulsation for such a nonhomogenizable acoustics is given by:

$$
\omega_{\mathrm{pd}}=O\left(\frac{\mu}{\rho_{F} l^{2}}\right)
$$

and we have:

$$
\omega_{f d} \ll \omega_{p v} \ll \omega_{p d} .
$$

The above analysis, which concerns double porosity media, can be used to interpret acoustical laboratory experiments on porous media where viscoelastic-like behaviours have been recorded (Murphy, 1982; Winkler and Nur, 1982; Jones and Nur, 1983). Many different explanations have been proposed to understand such behaviours but none of them is satisfying. A good review of them can be found in the book by Bourbie et al. (1986). In fact it is possible to show that a single porosity sample behaves like a slab of the micro-porous medium described in Section 4, when it is submitted to a pulsation $\omega_{p v}$.

We consider a cylindrical sample of a single porous medium with its axis in the $x^{\prime \prime}$ direction, saturated by and immersed in a viscous fluid. Different boundary conditions would lead to similar effects. For the sake of simplicity we adopt the above notations of the double porosity medium: the prime characterizes the porous medium and the double prime is for the macroscopic level. The sample is subjected to an acoustic excitation in the $x^{\prime \prime}$ direction, with a wavelength $l^{\prime \prime}$. The characteristic transverse size of the sample is denoted $l^{\prime}$. For most of the porous medium we have:

$$
\frac{l^{\prime}}{l^{\prime \prime}}=\varepsilon \ll 1
$$

Let us assume the pulsation of the excitation to be of the order of $\omega_{\mathrm{pv}}$. Therefore, the behaviour of the pore fluid is quasi-static and $\mathbf{K}_{\mathrm{p}}=\left\langle\mathbf{k}_{\mathrm{p}}\right\rangle_{\mathbf{\Omega}}$, is the classical noncomplex permeability. The dynamics appears in the bulk momentum balance, only. At the sample scale, because of the very long wavelength, the pressure in the external fluid does not depend on the local space variables $y^{\prime}=y^{\prime \prime} / \varepsilon$ and $z^{\prime}=z^{\prime \prime} / \varepsilon$; it is noted $p_{f}\left(x^{\prime \prime}\right)$. The description of the porous medium submitted to a constant pulsation excitation is given by:

$$
\begin{aligned}
& \nabla\left(\mathbf{K}_{\mathrm{p}} \Delta p\right)=\alpha^{\prime} i \omega \mathbf{e}\left(\mathbf{u}_{s}\right)+\beta^{\prime} i \omega p, \\
& \nabla\left(\mathbf{c}^{\prime} \mathbf{e}\left(\mathbf{u}_{s}\right)-\alpha^{\prime} p\right)=-\omega^{2}(l-n) \rho_{s} u_{s}+i \omega \rho_{F} \mathbf{v}_{p}
\end{aligned}
$$

in the porous medium and:

$$
\left(\mathbf{c}^{\prime} \mathbf{e}\left(\mathbf{u}_{s}\right)-\alpha^{\prime} p\right) \mathbf{N}^{\prime}=-p_{f} \mathbf{N}^{\prime}, \quad p=p_{f}\left(\mathbf{x}^{\prime \prime}\right)
$$


on the lateral boundary of the sample. Taking into account the order of magnitude of the pulsation, the above set is made dimensionless by using $l^{\prime \prime}$ as the characteristic lenght:

$$
\begin{aligned}
& \nabla\left(\mathbf{K}_{p} \nabla p\right)=\varepsilon^{-2} \alpha^{\prime} i \omega \mathbf{e}\left(\mathbf{u}_{s}\right)+\varepsilon^{-2} \beta^{\prime} i \omega p, \\
& \nabla\left(\mathbf{c}^{\prime} \mathbf{e}\left(\mathbf{u}_{s}\right)-\alpha^{\prime} p\right)=-\omega^{2}(l-n) \rho_{s} \mathbf{u}_{s}+i \omega \rho_{F} \mathbf{v}_{p}
\end{aligned}
$$

in the porous medium and:

$$
\left(\mathbf{c}^{\prime} \mathbf{e}\left(\mathbf{u}_{s}\right)-\alpha^{\prime} p\right) \mathbf{N}^{\prime}=-p_{f} \mathbf{N}^{\prime}, \quad p=p_{f}\left(\mathbf{x}^{\prime \prime}\right)
$$

on the lateral boundary of the sample. Introducing in this set the asymptotic developments in the form:

$$
\begin{aligned}
& \mathbf{u}_{s}=\mathbf{u}^{0}\left(x^{\prime}, y^{\prime}, z^{\prime}, x^{\prime \prime}\right)+\varepsilon \mathbf{u}^{l}\left(x^{\prime}, y^{\prime}, z^{\prime}, x^{\prime \prime}\right)+\cdots, \\
& \mathbf{v}_{p}=\mathbf{v}^{0}\left(x^{\prime}, y^{\prime}, z^{\prime}, x^{\prime \prime}\right)+\varepsilon \mathbf{v}^{1}\left(x^{\prime}, y^{\prime}, z^{\prime}, x^{\prime \prime}\right)+\cdots \\
& p=p^{0}\left(x^{\prime}, y^{\prime}, z^{\prime}, x^{\prime \prime}\right)+\varepsilon p^{l}\left(x^{\prime}, y^{\prime}, z^{\prime}, x^{\prime \prime}\right)+\cdots
\end{aligned}
$$

with $x^{\prime}=x^{\prime \prime} / \varepsilon, y^{\prime}=y^{\prime \prime} / \varepsilon, z^{\prime}=z^{\prime \prime} / \varepsilon$, it is easy to check that we obtain successive boundary value problems similar to those of Section 4 . Therefore, the acoustics of the sample is given by the Equations (48)-(50). It shows the viscoelastic-like behaviour which is observed during laboratory experiments. Consider, for example, a sample of Berea sandstone with the following characteristics:

$$
\mathbf{c}^{\prime}=3.57 \times 10^{10} \mathrm{~Pa}, \quad \mathrm{~K}_{\mathrm{p}}=5 \times 10^{-10} \mathrm{~m}^{2} \mathrm{~Pa}^{-1} \mathrm{~s}^{-1}, \quad \alpha^{\prime} \approx 1
$$

Laboratory experiments show a characteristic frequency about $3 \mathrm{kHz}$ (Winkler and Nur, 1982). From the above evaluation it is found that it corresponds to a quite plausible order of magnitude of the sample size $l^{\prime} \approx 3 \mathrm{~cm}$ : the samples investigated by Winkler and Nur had rectangular cross-sections $2.54 \times 2.54 \mathrm{~cm}^{2}$.

On the contrary to the double porosity medium, the acoustics of a single porosity medium is generally homogenizable when excitated by a pulsation $\omega_{p d}$. Therefore, the inverse of the attenuation in the single porosity sample will present two very well separated peaks at the two characteristic frequencies $\omega_{p v}$ and $\omega_{p d}$. Remark that changing the transverse size or the shape of the sample results in a change of the values of the coefficients in Equations (48)-(50). In the case of a single porosity medium, this macroscopic description is specific to a particular sample and it is not intrinsic to the porous material. The phenomenon is not homogenizable (Auriault, 1991a).

\section{References}

Auriault J.-L., 1980, Dynamic behaviour of a porous medium saturated by a newtonian fluid, Int. $J$. Engng. Sci. 18, 775-785.

Auriault J.-L., 1991a, Heterogeneous medium. Is an equivalent macroscopic description possible? Int. $J$. Engng. Sci. 29, 7, 785-795.

Auriault J.-L., 1991b, Dynamic behaviour of porous media, in J. Bear and M. Y. Corapcioglu (eds),

Transport Processes in Porous Media, Kluwer Academic Publishers, Dordrecht. 
Auriault J.-L., 1992, Acoustics of three phase porous media, in M. Quintard and M. Todorovic (eds), Heat and Mass Transfer, Elsevier, New York, pp. 221-224.

Auriault J. L. and Boutin C., 1992, Deformable porous media with double porosity. Quasi-statics. I, Coupling effects, Transport in Porous Media 7, 63-82.

Auriault J. L. and Boutin C., 1993, Deformable porous media with double porosity. Quasi-statics. II: Memory effects, Transport in Porous Media, 10, 153-169.

Biot M. A., 1941, General theory of three-dimensional consolidation, J. Appl. Physics 12, 155-164.

Biot M. A., 1956, Theory of propagation of elastic waves in a fluid-saturated porous solid. II Higher frequency range, J. Acous. Soc. Am. 28, 2, 179-191.

Borne L., 1983, Contribution à l'étude du comportement dynamique des milieux poreux saturés déformables. Etude de la loi de filtration dynamique, Doctorat thesis, Grenoble.

Bourbié T., Coussy O., and Zinszner B., 1986, Acoustique des milieux poreux, Editions Technip, Paris.

Jones T. and Nur A., 1983, Velocity and attenuation in sandstone at elevated temperatures and pressures, Geophys. Res. Lett. 10(2), 140-143.

Levy T., 1979, Propagation of waves in a fluid-saturated porous elastic solid, Int. J. Engng. Sci. 17, 1005-1014.

Murphy W. F., 1982, Effects of partial water saturation on attenuation in Massilon sandstone and Vycor porous glass, J. Acoust. Soc. Amer. 71, 6, 1458-1468.

Winkler K. W. and Nur A., 1982, Seismic attentuation: effects of pore fluids and frictional sliding, Geophysics 47, 1-15. 